\title{
EL PENSAMIENTO ETNOGRÁFICO Y ARQUEOLÓGICO DECIMONÓNICO COMO DISTORSIÓN DEL PASADO: LAS COMUNIDADES CAZADORAS-RECOLECTORAS COSTERAS
}

\author{
Nineteenth-century ethnographic and archaeological thinking as a distortion \\ of the past: coastal gatherer and hunting communities
}

Luis Blas CuAdRAdo-Martín

Recibido: 26/12/2017

Revisado: $12 / 03 / 2018$
Aceptado: $31 / 05 / 2018$

Publicado: 29/06/2018

\section{RESUMEN}

En el presente artículo se expone la influencia que los enfoques teóricos etnográficos decimonónicos han tenido en el campo disciplinar de la arqueología prehistórica hasta bien entrado el siglo XX; y cómo este hecho ha aportado una heurística y una hermenéutica sesgada en relación con el estudio y comprensión de las comunidades cazadorasrecolectoras costeras. Asimismo, se pone en valor la importancia de las corrientes revisionistas que surgieron a partir de 1980 y que iniciaron un proceso de desconstrucción teórica que ha proporcionado un marco de interpretación menos distorsionador sobre las citadas comunidades.

Palabras Clave

Adaptaciones acuáticas; Cazadores y recolectores costeros; Etnocentrismo; Revisionismo teórico.
ABSTRACT

This article explains the influence that nineteenth-century ethnographic theoretical approaches have exerted on prehistoric archaeology until well into the $20^{\text {th }}$ century. It also explains how that fact has provided a biased heuristic and hermeneutics with regards to the study and understanding of coastal gatherers and hunting communities. Likewise, there is a recognizable importance of the revisionist tendencies appearing after 1980 which initiated a process of theoretical deconstruction. This process has made possible a more accurate frame of interpretation of such communities.

\section{KEY WORDS}

Aquatic adaptations; Coastal hunter-gatherers communities; Ethnocentrism; Theoretical revisionism.

luis.b.cuadrado@gmail.com 


\section{INTRODUCCIÓN}

No se posee información "neutral" sobre comunidades cazadoras-recolectoras costeras de carácter prístino, ya que estas fueron las primeras en entrar en contacto con los europeos y padecer un proceso de aculturación donde su desarrollo cultural fue interrumpido y transformado (Bailey y Milner, 2002, 3). Por otro lado, los planteamientos antropológicos realizados en el siglo XIX por los primeros viajeros europeos y etnógrafos sobre las formaciones cazadoras-recolectoras costeras propiciaron un contexto teórico negativo que aportaron ideas, creencias y explicaciones poco fundadas $y$ totalmente mediatizadas por concepciones etnocentristas (Bailey, 2004, 41). A todo ello habría que añadirle las predilecciones de los arqueólogos por seleccionar aquellas analogías etnográficas más acordes con las preferencias del momento (Bailey y Milner, 2002, 3).

Por desgracia, este es el único tipo de información etnográfica e histórica registrada durante el siglo XIX y buena parte del XX sobre las comunidades cazadoras-recolectoras costeras.

\section{AnÁlisis de los teXtos DE DARWin}

Un ejemplo lo tenemos en las descripciones realizadas por Charles Darwin (1809-1882) en el viaje de exploración del bergantín HMS Beagle sobre el modo de vida costero:

«Nos preguntamos muchas veces qué goces puede proporcionar la vida a ciertos animales inferiores; icon cuánta mayor razón no podríamos preguntárnoslo respecto de estos salvajes! Por la noche, cinco o seis de estos seres humanos, desnudos y apenas protegidos contra el viento y la lluvia de este país terrible, se acuestan en el suelo húmedo apretados los unos contra los otros y encogidos como animales. Al bajar la marea, en invierno y en verano, de día y de noche, tienen que levantarse para ir a buscar conchas entre las rocas; las mujeres se sumergen para proporcionarse huevos de mar o permanecer horas enteras sentadas en las canoas hasta que logran pescar algunos pececillos con telas sin anzuelo. Si consiguen matar una foca o descubren el esqueleto medio podrido de una ballena, tienenlo por inmenso festín; se atracan de este innoble alimento, y para completar la fiesta comen algunas bayas o algunas setas que no saben a nada.
Con mucha frecuencia padecen hambres estos fueguenses" (Darwin, 2003, 442 y 443).

Este texto se complementa perfectamente con el siguiente:

« ¿Hay, por lo demás, en su existencia nada que pueda desarrollar facultades intelectuales elevadas? ¿Necesitan imaginación, razón, ni juicio? Nada tienen que imaginar, nada que comparar, nada que decidir. Para despegar una lapa de las piedras, ni aun necesita emplear la astucia, esa ínfima facultad del espíritu» (Darwin, 2003, 447 y 448).

Como podemos comprobar la valoración de desprestigio que Charles Darwin otorga en estos textos a toda una serie de estrategias económicas basadas en las adaptaciones acuáticas (recolección de moluscos intermareales, pesca de bajura de ictiofauna de poco tamaño, actividades cinegéticas sobre mamíferos marinos, captación de animales varados en las costas) corresponde al enfoque etnocéntrico singular y característico del siglo XIX.

Asimismo, la interpretación, anteriormente reseñada, se debe contextualizar en relación con el siguiente planteamiento antropológico:

«Darwin creía que los pueblos menos civilizados eran también los menos desarrollados intelectual y emocionalmente con respecto a los europeos; por tanto, su estimación sobre el desarrollo biológico se correspondía con la supuesta escala de evolución cultural» (Trigger, 1992, 113).

\section{Arqueología y Antropología}

Las visiones etnocéntricas indicadas traspasaron la etapa de fin de siglo llegando a afectar a la arqueología del siglo XX y generando, incluso, implicaciones y derivaciones ideológicas de tipo clasista. Estos aspectos se reflejan fielmente en la opinión emitida por el arqueólogo alemán Max Uhle (1856-1944) a principio del siglo XX:

" $[\ldots]$ the manner of procuring the essentials of life by collecting shells in itself indicates a low form of human existence. In all parts of the world, even today, people may be seen on the shore at low water gathering for food the shells uncovered by the retreating tide; and although under the changed conditions of life they raise no shell mounds, these people always belong to 
the lower classes of society, and lead in this manner a primitive as well as a simple life» (Uhle, 1907, 31).

Posteriormente, en la segunda mitad del siglo XIX, el antropólogo evolucionista norteamericano Lewis Henry Morgan (1818-1881) empleó un esquema evolucionista (Harris, 1985, 156) dividido en tres períodos étnicos: salvajismo, barbarie y civilización; los dos primeros a su vez se subdividían en inferior, medio y superior (véase Morgan 1987, 77-89). Asimismo, cada una de las etapas indicadas se definió en función de una serie de parámetros o innovaciones culturales.

Esta clasificación estaba bastante relacionada con las edades sucesivas de la Piedra, del Bronce y del Hierro determinadas por el investigador danés Christian Jürgensen Thomsen (1788-1865) (Harris, $1985,129)$, cuyos resultados fueron divulgados en 1836. La causa de este fenómeno deriva del compromiso que adoptaron la arqueología prehistórica y la etnología con el enfoque evolucionista unilineal (Trigger, 1992, 110).

En relación con el período étnico del salvajismo medio Lewis Henry Morgan determinaba lo siguiente:

"Comenzó con la adquisición de una subsistencia a base de pescado y el conocimiento del uso del fuego, y terminó con la invención del arco y la flecha» (Morgan, 1987, 82)

Como se puede deducir de la cita anterior las innovaciones culturales relacionadas con las adaptaciones acuáticas fueron encuadradas, según Morgan, en el nivel medio del salvajismo dentro de la escala de evolución cultural. Este posicionamiento producía, en función de la terminología utilizada, una connotación de inferioridad a las adaptaciones acuáticas. En el salvajismo medio situó a título de ejemplo paradigmático "a los australianos y la mayor parte de los polinesios, al tiempo de ser descubiertos" (Morgan, 1987, 82). En referencia al estadio superior del salvajismo los ejemplos seleccionados fueron las tribus Athapascan (bahía del Hudson), tribus del valle de Colombia y otras tribus pertenecientes a la costa de América del Norte y del Sur: todas ellas con relación a la época de su descubrimiento (Morgan, 1987, 82 y 83). En otras palabras: pueblos oceánicos como los polinesios y determinadas tribus costeras de América se incluyeron en los niveles "inferiores" (salvajismo) de la escala de la evolución cultural, con todas las consecuencias arqueológicas que este hecho conllevó durante el siglo XIX (véase también conclusiones de Bailey y Milner, 2002, 3 y 4; Bailey, 2004, 41). Aunque estas cuestiones se puedan encuadrar dentro de una perspectiva negativa, sí es cierto que para Morgan las estrategias de pesca tuvieron un papel importante como táctica de subsistencia:

«El pescado debe ser reconocido como la primera clase de alimentación artificial, desde que no era completamente aprovechable sin ser cocinado. No es improbable que el fuego tuviese su primer empleo para este fin. Los peces eran universales en su distribución, ilimitados en cantidad, y la única clase de alimento que podía obtenerse en todo tiempo» (Morgan, 1987, 91).

Es decir: interpretaba que las zonas acuáticas eran una verdadera cornucopia de provisiones (también Sauer, 1962, 45), un "Jardín del Edén» (véase Erlandson, 1994, 273) al que no le afectaba nunca la carestía y la falta de abastecimientos.

Todos estos estudios etnológicos influyeron de manera decisiva en la arqueología prehistórica, debido al posicionamiento común que ambas disciplinas tuvieron en Europa Occidental y Estados Unidos durante las décadas de los sesenta y los setenta (Trigger, 1992, 110). Otra razón para comprender la alineación mencionada está relacionada con la escasez de datos proporcionados por el registro arqueológico, lo cual indujo que los arqueólogos tuvieran que acudir a lingüistas, antropólogos físicos y, por supuesto, etnólogos para llevar a cabo las reconstrucciones históricas (Daniel, 1950,185 y 186) a través del denominado «método comparativo".

Esta postura teórica fue defendida por el prehistoriador británico John Lubbock, lord Avebury, (1834-1913). Para Lubbock el estudio de los primitivos contemporáneos de su época podría ilustrar, e incluso reconstruir, la cultura de los hombres prehistóricos (Harris, 1985, 130; Trigger, 1992, 115). La justificación de este método la derivó de la disciplina de la geología, concretamente de la paleontología de Charles Lyell (1797-1875). La analogía metodológica la planteaba Lubbock $(1865,416)$ de la siguiente manera: los artefactos de piedra y hueso de las culturas pretéritas son para 
el arqueólogo como los restos de animales extintos para el geólogo. Así llegó incluso a exponer explícitamente que los pueblos de Tasmania y de Sudamérica representan para el arqueólogo lo que el perezoso y la zarigüeya para el geólogo.

De esta manera, debido a la aplicación del método comparativo, John Lubbock trasladó las analogías etnográficas a la prehistoria europea, además de las nociones difundidas por Morgan (1877) de bajo nivel cultural (salvajismo); en este sentido, estableció una cierta correlación "entre los fueguinos y el pueblo sin nombre que había dado lugar a los vertederos daneses mesolíticos, aunque notó que estos últimos habían superado a los fueguinos, ya que manufacturaron cerámica cruda" (Trigger, 1992. 115). Asimismo, también promulgó que en la Europa Mesolítica los grupos cazadoresrecolectores se tuvieron que "refugiar" en zonas acuáticas como las riberas de los ríos, orillas de los lagos o zonas costeras, debido a que se encontraban en una etapa de desarrollo caracterizada por un "bajo nivel cultural” (Bailey y Milner 2002, 4; Bailey, $2004,41)$. Este planteamiento de origen etnográfico, donde se equiparaba zona acuática con grupos de cultura inferior (véase también Bailey, 1975, 58), permaneció inalterable y con plena vigencia en los estudios prehistóricos sobre el Mesolítico hasta mediados del siglo XX (Bailey y Milner, 2002, 4; Bailey, 2004, 41), como se puede comprobar, de manera explícita, en el siguiente texto extraído de la obra clásica titulada Prehistoric Europe: The Economic Basis de Grahame Clark (1952):

«On the other hand a diet in which Shellfish are a mainstay is normally associated with a low level of culture, a proposition which is hardly contradicted by the attainments of the Capsians of the Tagus Valley, the Tardenoisians of the islands off Morbihan, the Obanians of Western Scotland or the ErtebØlle people of the Litorina coasts of Denmark» (Clark, 1952, 63).

El modelo etnográfico expuesto dominó los enfoques heurísticos de investigación destinados al estudio y comprensión de los recursos acuáticos (e.g. Cohen, 1981, 91) y de las sociedades cazadoras, recolectoras y pescadoras durante un período importante del siglo XX. En la década de 1980 se inició un proceso revisionista de rehabilitación y reinterpretación de los recursos y comunidades mencionadas (e.g. Yesner, 1980; Rowley-Conwy,
1983; Renouf, 1984; 1988) a partir de una serie de estudios que modificaron sustancialmente los condicionantes y limitaciones ideológicas que se tenían sobre las mismas. Así, se ha constatado que, aunque no todas, una serie de sociedades cazadorasrecolectoras costeras posglaciares tendieron hacia la aparición de la complejidad cultural, estableciendo parámetros arqueológicos caracterizados por el sedentarismo o semi-sedentarismo, alta densidad de población, sistemas de almacenamiento, especialización tecnológica y jerarquización social (Yesner, 1980; Rowley-Conwy, 1983; Renouf, 1984, 19; Bailey y Parkington, 1988, 9). Estos elementos culturales se encuentran muy cercanos al desarrollo de las sociedades agrícolas incipientes (Bailey y Milner, 2002, 2; Bailey, 2004, 41) y han podido estimular el desarrollo de sociedades de nivel estatal (e.g. Moseley, 1975). El investigador Jon M. Erlandson $(1994,1)$ incluso defiende una estrecha relación y continuidad entre el registro etnohistórico y etnográfico proporcionado por las comunidades cazadoras-recolectoras de la costa californiana del tiempo del contacto europeo, caracterizadas por la densidad de población y la complejidad cultural, y los datos ofrecidos por el registro arqueológico de California. Este enlace entre presente y pasado nos induce a pensar que a lo mejor esta era la regla en el pasado y no la excepción (Yesner, 1980, 728). En este sentido, hay que reseñar lo siguiente:

«[... los grupos cazadores y recolectores contemporáneos son anómalos debido simplemente al mero hecho de que siguen siendo cazadores y recolectores [...] Evidentemente, esos grupos están preseleccionados por su conservadurismo tecnológico» (Cohen, 1981, 60).

\section{Conclusiones}

Como se ha podido evidenciar las aportaciones teóricas etnográficas y arqueológicas han condicionado de forma negativa la percepción pretérita de las comunidades cazadoras-recolectoras costeras. Este hecho ha derivado en un enfoque sesgado de las citadas actividades, lo cual ha provocado una importante distorsión sobre las capacidades adaptativas de las mismas y su influencia en el desarrollo de los diferentes parámetros que definen el fenómeno de la complejidad social. Asimismo, los enfoques etnológicos y los correspondientes a la arqueología prehistórica, han 
infravalorado las capacidades neurocognitivas y motoras necesarias para la captura, manipulación y consumo de los recursos acuáticos, insertándolos siempre en estadios de bajo nivel cultural, como fue el caso del Mesolítico hasta mediados del siglo XX.

$\mathrm{La}$ introducción por derecho propio de las sociedades cazadoras, recolectoras y pescadoras en la investigación prehistórica ha sido un acontecimiento reciente en el tiempo (Bailey, 2004, 41). Este hecho ha sido impulsado por corrientes revisionistas, que en las últimas décadas han conseguido superar la minusvaloración y aislamiento al que habían sometido a los grupos humanos costeros.

\section{REFERENCIAS}

Bailey, G.N.(1975), "The Role of Shellfish in Coastal Economies: The Results of Midden Studies in Australia”, Journal of Archaeological Science, 2, 45-62.

Bailey, G.N. (2004), "World Prehistory from the Margins: the Role of Coastlines in Human Evolution", Journal of Interdisciplinary Studies in History and Archaeology, 1, 39-50.

Bailey, G.N. y Milner, N. (2002), "Coastal huntergatherers and social evolution: marginal or central?" Before Farming: the Archaeology of Old World Hunter-Gatherers, 3/4, 1-22.

Bailey, G. N. y Parkington, J. (1988), "The archaeology of prehistoric coastlines: an introduction", The Archaeology of Prehistoric Coastlines, (Bailey, G. y Parkington, J. Eds.), Cambridge, 1-10.

Clark, J.G.D. (1952), Prehistoric Europe: The Economic Basis, Londres.

Cohen, M.N. (1981), La crisis alimentaria de la prehistoria, Madrid.

Daniel, G.E. (1950), A Hundred Years of Archaeology, Londres.

Darwin, C. (2003), Viaje de un naturalista alrededor del mundo, Santa Fe. [Original: 1839, Journal of Researches into the Natural History and Geology of the Countries Visited during the Voyage Round the World of H.M.S. "Beagle" Under Command of Captain Fitz Roy, R.N. Londres].

Erlandson, J.M. (1994), Early Hunter-Gatherers of the California Coast, Nueva York.

Harris, M. (1985), El desarrollo de la teoría antropológica. Una historia de las teorías de la cultura, Madrid.
Lubbock, J. (1865), Pre-historic Times, as Illustrated by Ancient Remains, and the Manners and Customs of Modern Savages, Londres.

Lyell, C. (1853), Principles of Geology; or, the Modern Changes of Earth and its Inhabitants, Londres.

Morgan, L.H. (1987), La sociedad primitiva, Madrid. [Original: 1877, Ancient Society, or Researches in the Lines of Human Progress from Savagery through Barbarism to Civilization, Londres].

Moseley, M.E. (1975), The Maritime Foundations of Andean Civilization, Menlo Park.

Renouf, M.A.P. (1984), "Northern Coastal HunterFishers: An Archaeological Model", World Archaeology, 16, 18-27.

Renouf, M.A.P. (1988), "Sedentary coastal hunterfishers: an example from the Younger Stone Age of northern Norway", The Archaeology of Prehistoric Coastlines, (Bailey, G. y Parkington, J. Eds.), Cambridge, 102-115.

Rowley-Conwy, P. (1983), "Sedentary hunters: The Ertebolle example", Hunter-Gatherer Economy in Prehistory, (Bailey, G.N. Eds.), Cambridge, 111-126.

Sauer, C.O. (1962), "Seashore-primitive home of man?" Proceedings of the American Philosophical Society, 106, 41-47.

Thomsen, C.J. (1836), "Kortfattet udsigt over mindesmærker og oldsager fra nordens oldtid", Ledetraad til Nordisk Oldkyndighed (Rafn, C.C. Ed.), Copenhague, 27-90.

Trigger, B.G. (1992), Historia del pensamiento arqueológico, Barcelona.

Uhle, M. (1907), "The Emeryville Shellmound", University of California Publications in American Archaeology and Ethnology,7, Berkeley, 1-107.

Yesner, D.R. (1980), "Maritime HunterGatherers. Ecology and Prehistory", Current Anthropology, 21, 727-735. 
